\title{
Prediction of post TIPS hepatic encephalopathy: are we there yet?
}

\author{
Sanchit Sharma ${ }^{1} \cdot$ Ashish Chauhan $^{2} \cdot$ Anoop Saraya $^{1} \mathbb{C}$ \\ Received: 14 May 2021 / Accepted: 25 May 2021 / Published online: 11 June 2021 \\ (c) Asian Pacific Association for the Study of the Liver 2021
}

\section{Dear Editor}

We read with great interest the article by Dr. Yang and colleagues about the excellent performance of pre-intervention prediction models to predict the risk of overt hepatic encephalopathy (HE) after transjugular intrahepatic portosystemic shunt (TIPS) insertion in patients with cirrhosis [1]. Authors have given an interesting proof of concept about the ensemble of clinical, biochemical, and imaging parameters to predict the risk of HE in these patients. We would like to highlight a few points relevant to this study.

This was a retrospective analysis of a database and had its inherent risks of selection bias. While these algorithms fit perfectly on the database, they carry a limited significance in the absence of their validation in a prospective data. Further, authors took various imaging parameters on the assumption that these demonstrate severity of liver disease and, hence, may predict the occurrence of post-TIPS HE. In the absence of their correlation with hepatic venous pressure gradient, elastography and outcomes, the role of these included parameters beyond demonstration of chronic liver disease (CLD) and presence of clinically significant portal hypertension (CSPH) has limited evidence. The same may not be relevant in present cohort as all these patients had CLD and CSPH. Interestingly, liver surface nodularity, a parameter which correlates with severity of CSPH and risks of decompensation, was not included in these prediction models $[2,3]$. Although, some parameters emerged significant on multivariate analysis, this association even though plausible, needs a mandatory validation on a different cohort before application of them for model generation.

Anoop Saraya

ansaraya@yahoo.com

1 Department of Gastroenterology and Human Nutrition Unit, All India Institute of Medical Sciences, New Delhi 110029, India

2 Department of Gastroenterology, Indira Gandhi Medical College, Shimla, Himachal Pradesh, India
Limited information is described regarding the outcomes of these patients after TIPS. The incidence of post-TIPS HE in this cohort is not presented in results which is the most important factor governing the performance of the models described by the authors. Similarly, it would be interesting to know other outcomes like transplant free survival, incidence of hepatocellular carcinoma and outcomes of other clinical decompensations. It is also important to know, if any of the included patients had any previous episodes of HE and whether this was incorporated in the cox proportional hazard model to estimate the risk of post-TIPS HE.

Funding We have no financial disclosures to make.

\section{Declarations}

Conflict of interest All authors (Sanchit Sharma, Ashish Chauhan and Anoop Saraya) have no conflicts of interest to declare.

\section{References}

1. Yang Y, Fu S, Cao B, et al. Prediction of overt hepatic encephalopathy after transjugular intrahepatic portosystemic shunt treatment: a cohort study. Hepatol Int 2021. https://doi.org/10.1007/ s12072-021-10188-5

2. Smith AD, Zand KA, Florez E, et al. Liver surface nodularity score allows prediction of cirrhosis decompensation and death. Radiology 2016;283(3):711-722. https://doi.org/10.1148/radiol. 2016160799

3. Sartoris R, Rautou P-E, Elkrief L, et al. Quantification of liver surface nodularity at CT: utility for detection of portal hypertension. Radiology 2018;289(3):698-707 https://doi.org/10.1148/radiol. 2018181131

Publisher's Note Springer Nature remains neutral with regard to jurisdictional claims in published maps and institutional affiliations. 